\title{
Towards an Efficient System for Differentially-private, Cross-device Federated Learning
}

\author{
Kunlong Liu, Richa Wadaskar, and Trinabh Gupta \\ University of California, Santa Barbara
}

\begin{abstract}
This paper describes fresh ideas for a new system for federated averaging that aims to efficiently provide differential privacy even when a fraction of devices is malicious and there is no trusted core.
\end{abstract}

Introduction. How can we efficiently run federated learning, particularly federated averaging (FedAvg) [13], over a large number of user devices, e.g., hundreds of thousands to several billion, while guaranteeing differential privacy $(D P)[7]$ to the device owners?

This question is motivated by two recent trends. First, FedAvg has found multiple use cases in industry. Google uses it to train a LSTM recurrent neural network for next-word prediction in Gboard (mobile keyboard) [9], and Mozilla uses it to train a ranking model for auto completion of suggestions in address bar [10]. Second, DP is a gold standard for privacy as it provides provable guarantees about an individual's data.

One approach to augment FedAvg with DP is to compose additively homomorphic encryption (AHE) with central DP (CDP) $[18,19]$. (An alternative is to use secure aggregation $[3,5]$, but we prefer the homomorphic encryption route as it has been shown to compose with zero-knowledge (ZK) proofs [4] to additionally guarantee correctness of training [15].) At a high level, devices upload encrypted gradients to an aggregator, who sums them using the homomorphic properties of the encryption, before adding noise for DP. CDP has shown to reach higher model accuracy over local DP [8], where each device sends noised gradients to the aggregator. However, it is challenging to build an efficient system with this approach at scale under a strong threat model where the devices or the aggregator may be malicious. For example, the aggregator can reveal a device's input by multiplying it with a large value such that the DP noise is insufficient.

Orchard $[15,16]$, a state-of-the-art system based upon $\mathrm{CDP}$, uses a mixture of cryptographic primitives such as MPC [11], AHE, and Merkle trees [14] to guarantee DP at scale even if the aggregator is occasionally byzantine and a small fraction of the devices are malicious. It also improves reliability of training by using ZK-proofs [4]. However, it

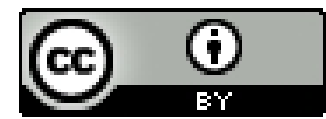

This work is licensed under a Creative Commons Attribution International 4.0 License. ResilientFL '21, October 25, 2021, Virtual Event, Germany

(c) 2021 Copyright held by the owner/author(s).

ACM ISBN 978-1-4503-8708-8/21/10.

https://doi.org/10.1145/3477114.3488762 is expensive for devices. If applied to the Gboard model containing $1.4 \mathrm{M}$ parameters (1.4 MiB in total size), Orchard would incur $764 \mathrm{MiB}$ of downloads and $\approx 4$ minutes of $\mathrm{CPU}$ time per device per round of training (the model takes 3,000 rounds to converge).

This paper discusses the initial design of a system, Atom, that improves these efficiency bottlenecks. For the Gboard model, we project, based on microbenchmarks, that Atom would reduce per-device downloads to less than $5 \mathrm{MiB}$ per round, use a device's CPU only for a few rounds of training, and move $\approx 1 / 3$ rd of the CPU time to an offline phase to reduce the overall system training time. We first present a brief overview of Orchard's threat model and bottlenecks, and then dive into Atom's improvements.

Overview of Orchard. Orchard consists of an aggregator and a large number of user devices, and assumes that they follow the $O B+M C$ threat model. The aggregator is honestbut-curious most of the time but may be occasionally byzantine $(\mathrm{OB})$, say when a rogue system administrator is logged in. The devices are mostly correct (MC), but a configurable small fraction (1-5\%) can be malicious. Even though this fraction is small, the absolute number of malicious devices is substantial (e.g., millions out of a billion devices).

At a high-level, Orchard follows the collect-and-verify approach, where in the collect phase the aggregator collects encrypted gradients and ZK-proofs from the devices, and in the verify phase the devices verify the aggregator's work, that it performed the summation correctly.

There are two key performance-related aspects of these phases. First, during collect, a device's computation time is dominated by the time to generate the ZK-proof that indicates gradients are within a specified range and their encryption was performed correctly. Second, during verify, a device's costs are dominated by network downloads. In more detail, after the collect phase, the aggregator adds the homomorphically encrypted gradients by forming a summation tree (encrypted gradients are arranged as leaf nodes of a tree, and the tree is traversed to its root while computing each parent ciphertext(s) as a sum of its children). During verify, each device downloads a part of the tree, specifically, a configurable number of $s+1$ consecutive leaf nodes and their $\lceil s / 2\rceil$ parent nodes, and $s$ random non-leaf nodes and their parents. The device then asserts that the parent ciphertexts equal the sum of their children.

Cost analysis: We empirically evaluated the main clientside components of Orchard, implementing the ZK-proof generation in 461 lines of Rust using ark-groth16 [2]. For the 
Gboard model, each device's input (i.e., a leaf node) is 342 ciphertexts, with 4,096 parameters in each ciphertext. On a 6-core, $2.6 \mathrm{GHz}$ laptop with $8 \mathrm{~GB}$ of RAM, it takes 235.1 s to generate the proofs for these ciphertexts. Each device also downloads $760 \mathrm{MiB}$ of data for tree verification; this includes 17 tree nodes each containing 342 ciphertexts. These costs are substantial especially as the plaintext model is small (1.4 MiB). Moreover, the total training time can be high: if a client had a bandwidth of $53 \mathrm{Mbps}$ [1], then downloading $760 \mathrm{MiB} 3 \mathrm{~K}$ times would itself take over 4 days.

Atom: Switching to stochastic FedAvg. A core reason Orchard incurs high costs is that the system is designed for fullbatch (non-stochastic) gradient descent: every device participates in each round of training and contributes a leaf node which is then checked for correctness. But full-batch gradient descent is not necessary. For example, fig. 1 shows training accuracy as a function of the number of training rounds for different number of participant devices per round for a DNN over the EMNIST dataset [6]

(containing 3,383

devices in total).

The training accu-

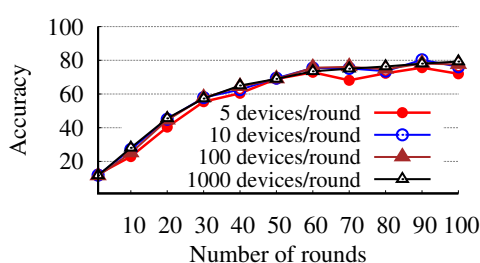

Figure 1: Non-private training accuracy. racy can be competitive even when a small fraction of devices contribute gradients in a round; this is the preferred stochastic gradient descent method.

Switching to stochastic FedAvg in Orchard requires technical work, as simply asking a subset of devices to upload gradients induces redundant verification work. For example, if the number of leaf nodes is $1 \mathrm{~K}$ (for $1 \mathrm{~K}$ devices participating in a round), and the total number of devices is $1 \mathrm{M}$, then many devices download and check the same nodes of the summation tree. Instead, Atom leverages the fact that leaf nodes are made of multiple ciphertexts (e.g., 342 for the Gboard model) that can be checked in parallel. To elaborate, after collecting encrypted gradients from participating devices, the aggregator forms $P$ summation trees, one for each ciphertext in the original leaf node. Then, during the verify phase, a device checks the $p$-th $(1 \leq p \leq P)$ tree with a certain probability that ensures that every node gets checked at least once by an honest device. The benefit is that each device's network downloads reduce approximately by the fraction of participants per round: for the Gboard model, each device downloads $11.24 \mathrm{MiB}$ per round, when $1 \%$ of total devices contribute gradients in a round.

Optimizing checking of ciphertext sums. After applying the above optimization, each device still downloads at least two leaf nodes and three non-leaf nodes for one summation tree. Atom reduces this cost by incorporating the polynomial

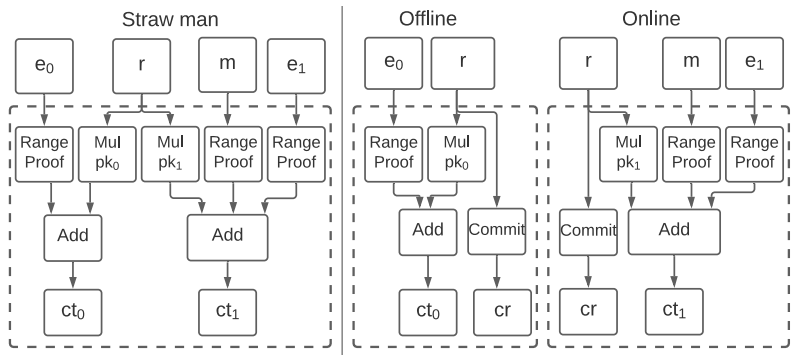

Figure 2: Encryption and its splitting into offline/online parts. $e_{0}, e_{1}, r$ are sampled from a polynomial ring, $m$ is an input message, $p k$ is a public key, $\left(c t_{0}, c t_{1}\right)$ is the ciphertext, and $c r$ is a commitment.

identity testing (PIT) $[17,20]$ : given a $d$-degree polynomial $f(x)$ over the field $\mathbb{F}_{p}$, one can test whether $f(x)$ is a zero polynomial by sampling $r \in \mathbb{F}_{p}$ uniformly and testing if $f(r)==0$. The intuition is that a $d$-degree polynomial has at most $d$ solutions to $f(x)==0$, and thus the probability of $f(r)==0$ for a non-zero $f(x)$ is at most $d /\left|\mathbb{F}_{p}\right|$.

Atom incorporates PIT by observing that Orchard uses a Ring-LWE AHE scheme [12], where each ciphertext is a pair of polynomials. Thus, the goal of summation checking is to test whether the polynomial $c_{\text {left-child }}(x)+c_{\text {right-child }}(x)-$ $c_{\text {parent }}(x)=\mathbf{0}$, where $c(\cdot)$ denotes a ciphertext. By applying the PIT, Atom transforms this check into checking whether $c_{\text {left-child }}(r)+c_{\text {right-child }}(r)-c_{\text {parent }}(r)==0$ at a random point $r$. The gain is that a device downloads two field elements rather than an entire ciphertext (8,192 field elements) for the non-leaf nodes of the summation tree, although it still downloads the ciphertexts at the leaf nodes to check that their evaluation at $r$ is what the aggregator claims. After incorporating PIT, a device's downloads reduce by $2.4 \times$ to 4.52 MiB for the Gboard model.

Splitting ZK-proof generation across rounds. Atom's switch to stochastic FedAvg frees devices from generating gradients in all rounds. Thus, a Atom device can move part of proof generation to an offline phase that runs before the round(s) in which it participates. The left part of figure 2 shows the original computation a device runs inside a ZKproof system. Atom takes the part of the computation that does not depend on the message being encrypted and runs it as an offline computation, and the rest as an online computation (the right part of Figure 2). Atom utilizes a commitment to ensure that a device uses the same common inputs across the offline and online phases.

For the Gboard model, Orchard's ZK-proof computation consumes 235.1s on a 6-core laptop, and Atom's computation consumes 141.9s and 155.6s for the offline and online phase, respectively. Though the total time in the offline/online scheme is higher due to the addition of the commitment, we argue that this trade-off is justified as devices contribute gradients in a few rounds (rather than all rounds), and the offline/online split reduces the overall system training time. 


\section{REFERENCES}

[1] Where do U.S. mobile speeds rank globally? https: //www.allconnect.com/blog/us-mobile-speeds-globally. Accessed: 2021-09-12.

[2] arkworks. ark-groth16. https://github.com/arkworks-rs/groth16.

[3] J. H. Bell, K. A. Bonawitz, A. Gascón, T. Lepoint, and M. Raykova. Secure single-server aggregation with (poly) logarithmic overhead. In ACM Conference on Computer and Communications Security (CCS), pages 1253-1269, 2020.

[4] E. Ben-Sasson, A. Chiesa, E. Tromer, and M. Virza. Succinct noninteractive zero knowledge for a von neumann architecture. In USENIX Security Symposium (SEC), pages 781-796, 2014.

[5] K. Bonawitz, V. Ivanov, B. Kreuter, A. Marcedone, H. B. McMahan S. Patel, D. Ramage, A. Segal, and K. Seth. Practical secure aggregation for privacy-preserving machine learning. In ACM Conference on Computer and Communications Security (CCS), pages 1175-1191, 2017.

[6] G. Cohen, S. Afshar, J. Tapson, and A. Van Schaik. EMNIST: Extending MNIST to handwritten letters. In International foint Conference on Neural Networks (IfCNN), pages 2921-2926, 2017.

[7] C. Dwork, F. McSherry, K. Nissim, and A. Smith. Calibrating noise to sensitivity in private data analysis. In Theory of Cryptography Conference (TCC), pages 265-284, 2006.

[8] Ú. Erlingsson, V. Pihur, and A. Korolova. RAPPOR: Randomized aggregatable privacy-preserving ordinal response. In ACM Conference on Computer and Communications Security (CCS), pages 1054-1067, 2014

[9] A. Hard, K. Rao, R. Mathews, S. Ramaswamy, F. Beaufays, S. Augenstein, H. Eichner, C. Kiddon, and D. Ramage. Federated learning for mobile keyboard prediction. arXiv preprint arXiv:1811.03604, 2018.

[10] F. Hartmann, S. Suh, A. Komarzewski, T. D. Smith, and I. Segall. Federated learning for ranking browser history suggestions. arXiv preprint arXiv:1911.11807, 2019.

[11] KU Leuven COSIC. SCALE-MAMBA. https://github.com/KULeuvenCOSIC/SCALE-MAMBA

[12] V. Lyubashevsky, C. Peikert, and O. Regev. On ideal lattices and learning with errors over rings. In Annual International Conference on the Theory and Applications of Cryptographic Techniques (EUROCRYPT), 2010 .

[13] B. McMahan, E. Moore, D. Ramage, S. Hampson, and B. A. y Arcas Communication-efficient learning of deep networks from decentralized data. In Artificial intelligence and statistics, pages 1273-1282, 2017.

[14] R. C. Merkle. A digital signature based on a conventional encryption function. In Annual International Conference on the Theory and Applications of Cryptographic Techniques (EUROCRYPT), pages 369-378, 1987.

[15] E. Roth, D. Noble, B. H. Falk, and A. Haeberlen. Honeycrisp: Largescale differentially private aggregation without a trusted core. In $A C M$ Symposium on Operating Systems Principles (SOSP), pages 196-210, 2019

[16] E. Roth, H. Zhang, A. Haeberlen, and B. C. Pierce. Orchard: Differentially private analytics at scale. In USENIX Symposium on Operating Systems Design and Implementation (OSDI), pages 1065-1081, 2020.

[17] J. T. Schwartz. Fast probabilistic algorithms for verification of polynomial identities. Fournal of the ACM ( $7 A C M), 27(4): 701-717,1980$.

[18] S. Truex, N. Baracaldo, A. Anwar, T. Steinke, H. Ludwig, R. Zhang, and Y. Zhou. A hybrid approach to privacy-preserving federated learning. In ACM Workshop on Artificial Intelligence and Security, pages 1-11, 2019.

[19] R. Xu, N. Baracaldo, Y. Zhou, A. Anwar, and H. Ludwig. Hybridalpha: An efficient approach for privacy-preserving federated learning. In ACM Workshop on Artificial Intelligence and Security, pages 13-23,
2019.

[20] R. Zippel. Probabilistic algorithms for sparse polynomials. In International symposium on symbolic and algebraic manipulation, pages 216-226, 1979 